Commun. Fac. Sci. Univ. Ank. Ser. A1 Math. Stat.

Volume 68, Number 2, Pages 1797-1810(2019)

DOI: $10.31801 /$ cfsuasmas. 481747

ISSN 1303-5991 E-ISSN 2618-6470

http://communications.science.ankara.edu.tr/index.php?series=A1

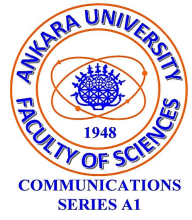

\title{
PRINCIPAL FUNCTIONS OF IMPULSIVE DIFFERENCE OPERATORS ON SEMI AXIS
}

\author{
ŞEYHMUS YARDIMCI AND IBRAHIM ERDAL
}

\begin{abstract}
In this paper, we investigate the continuous spectrum and resolvent operator of a second-order difference operator with an impulsive condition. Then, under certain conditions, we prove finiteness of eigenvalues, spectral singularities. At last, we present principal functions of corresponding impulsive operator.
\end{abstract}

\section{INTRODUCTION}

Researchers often encounter some discontinuities or degenerations during many evolution processes. At a certain moment, the state may change abruptly and takes a short time compared to the whole duration. These sudden effects are recognized as instantaneous impulses. The models involving impulsive effects are called impulsive equations. There are great contributions in [1, 2, 3, to the theory of impulsive differential equations. The mathematical or physical models concerning such impulses are also called the equations with a transmission effect, or the equations with a point interaction [4, 5. Over the years, some results arising from impulsive effects were carried over quite easily to the discrete case. 6, 7, 8, are outstanding studies on impulsive difference equations.

The main equation we investigate in this paper is

$$
a_{n-1} y_{n-1}+b_{n} y_{n}+a_{n} y_{n+1}=\lambda y_{n}, \quad n \in \mathbb{N},
$$

which is the discrete analogue of the Sturm-Liouville equation

$$
-y^{\prime \prime}+q(x) y=\lambda^{2} y, \quad x \in[0, \infty),
$$

where $\lambda$ is a spectral parameter, $\left\{a_{n}\right\}_{n \in \mathbb{N} \cup\{0\}},\left\{b_{n}\right\}_{n \in \mathbb{N}}$ are complex sequences and $q$ is a complex valued function. Naimark [9] initiated the study of continuous and discrete spectrum of Sturm-Liouville operator corresponding to 2 with a boundary condition $y(0)=0$. In addition to [9], we refer to [10, 11] for further information

Received by the editors: November 12, 2018; Accepted: April 29, 2019.

2010 Mathematics Subject Classification. 34L05, 34L15, 39A13, 39A70, 47B39.

Key words and phrases. Impulsive condition, difference operator, spectrum, principal functions.

(C)2019 Ankara University Communications Faculty of Sciences University of Ankara-Series A1 Mathematics and Statistics 
on spectral theory of Sturm-Liouville equations. On the other hand, for difference equations, a lot of spectral results have been investigated in the literature [12, 13. Moreover, for the spectral theory of difference equations, [14, 15, 16, 17, 18, 19, 20] are detailed references for the readers. But these references are all related to general boundary conditions. Hence, the aim of this paper is to study some spectral properties of the impulsive difference operator mentioned in [20] which is still an uninvestigated problem in literature.

We shall consider the following second-order difference equation

$$
a_{n-1} y_{n-1}+b_{n} y_{n}+a_{n} y_{n+1}=\lambda y_{n}, \quad n \in \mathbb{N} \backslash\{k-1, k, k+1\}
$$

with the boundary condition

$$
y_{0}=0
$$

and the impulsive condition

$$
\left(\begin{array}{c}
y_{k+1} \\
\Delta y_{k+1}
\end{array}\right)=B\left(\begin{array}{c}
y_{k-1} \\
\nabla y_{k-1}
\end{array}\right), B=\left(\begin{array}{cc}
\alpha & \beta \\
\gamma & \delta
\end{array}\right)
$$

where $\lambda=2 \cos z$ is a spectral parameter, $\alpha, \beta, \gamma, \delta$ are complex numbers, $\nabla$ denotes the backward difference operator and $\Delta$ denotes the forward difference operator, i.e.,

$$
\begin{aligned}
\nabla y_{n}: & =y_{n}-y_{n-1} \\
\Delta y_{n} & :=y_{n+1}-y_{n}
\end{aligned}
$$

Throughout the paper, we assume that $a_{n} \neq 0$, for all $n \in \mathbb{N} \cup\{0\},\left\{a_{n}\right\}_{n \in \mathbb{N} \cup\{0\}}$ and $\left\{b_{n}\right\}_{n \in \mathbb{N}}$ are complex sequences satisfying the condition

$$
\sum_{n \in \mathbb{N}} n\left(\left|1-a_{n}\right|+\left|b_{n}\right|\right)<\infty
$$

Without impulsive condition (5), equation (3) has the bounded solution satisfying the condition

$$
\lim _{n \rightarrow \infty} e^{-i n z} e_{n}(z)=1 \text {, }
$$

for $\lambda=2 \cos z$, where $z \in \overline{\mathbb{C}}_{+}:=\{z \in \mathbb{C}: \operatorname{Im} z \geq 0\} \cdot e_{n}(z)$ is called the Jost solution of (3). It is analytic with respect to $z$ in $\mathbb{C}_{+}:=\{z \in \mathbb{C}: \operatorname{Im} z>0\}$, continuous in $\overline{\mathbb{C}}_{+}$and $e_{n}(z+2 \pi)=e_{n}(z)$ for all $z$ in $\overline{\mathbb{C}}_{+}$. Besides, the function $e_{n}(z)$ has the representation $[17]$

$$
e_{n}(z)=\mu_{n} e^{i n z}\left(1+\sum_{m=1}^{\infty} A_{n m} e^{i m z}\right), \quad n \in \mathbb{N},
$$

where $\mu_{n}$ and $A_{n m}$ are expressed in terms of the sequences $\left\{a_{n}\right\}_{n \in \mathbb{N} \cup\{0\}}$ and $\left\{b_{n}\right\}_{n \in \mathbb{N}}$ as

$$
\begin{aligned}
\mu_{n} & =\left\{\prod_{k=n}^{\infty} a_{k}\right\}^{-1}, \\
A_{n 1} & =-\sum_{k=n+1}^{\infty} b_{k},
\end{aligned}
$$




$$
\begin{aligned}
A_{n 2} & =\sum_{k=n+1}^{\infty}\left\{\left(1-a_{k}^{2}\right)+b_{k} \sum_{s=k+1}^{\infty} b_{s}\right\}, \\
A_{n, m+2} & =A_{n+1, m}+\sum_{k=n+1}^{\infty}\left\{\left(1-a_{k}^{2}\right) A_{k+1, m}-b_{k} A_{k, m+1}\right\}, m=1,2, \ldots ; n \in \mathbb{N} .
\end{aligned}
$$

Moreover, $A_{n m}$ satisfies

$$
\left|A_{n m}\right| \leq c \sum_{k=n+\left[\frac{m}{2}\right]}^{\infty}\left(\left|1-a_{k}\right|+\left|b_{k}\right|\right),
$$

where $c>0$ is a constant and $\left[\frac{m}{2}\right]$ denotes the integer part of $\frac{m}{2}$.

On the other hand, two solutions of impulsive difference boundary value problem (3) - (5) are stated in [20] as

$$
E_{n}(z)=\left\{\begin{array}{cc}
\frac{M_{22}}{\operatorname{det} M} Q_{n}(z)-\frac{M_{21}}{\operatorname{det} M} P_{n}(z), & n=0,1,2, \ldots, k-1 \\
e_{n}(z), & n=k+1, k+2, \ldots
\end{array}\right.
$$

and

$$
F_{n}(z)=\left\{\begin{array}{cc}
P_{n}(z), & n=0,1,2, \ldots, k-1 \\
M_{12} e_{n}(z)+M_{22} e_{n}(-z), & n=k+1, k+2, \ldots
\end{array},\right.
$$

where $Q_{n}(z)$ and $P_{n}(z)$ are the fundamental solutions of (3) satisfying

$$
\begin{array}{ll}
Q_{0}(z)=\frac{1}{a_{0}}, & Q_{1}(z)=0 \\
P_{0}(z)=0, & P_{1}(z)=1 .
\end{array}
$$

Remember that $e_{n}(-z)$ is another solution of (3) fulfilling the asymptotic condition

$$
\lim _{n \rightarrow \infty} e^{i n z} e_{n}(-z)=1, \quad z \in \overline{\mathbb{C}}_{-}:=\{z \in \mathbb{C}: \operatorname{Im} z \leq 0\},
$$

and coefficients $M=\left[M_{i j}\right]_{2 x 2} i, j=1,2$ is defined as transfer matrix $M$ such that

$$
\begin{aligned}
M_{22}(z)=-\frac{a_{k+1}}{2 i \sin z}\left\{-\Delta e_{k+1}(z)[\right. & \left.\alpha P_{k-1}(z)+\beta \nabla P_{k-1}(z)\right] \\
& \left.+e_{k+1}(z)\left[\gamma P_{k-1}(z)+\delta \nabla P_{k-1}(z)\right]\right\}, \\
M_{12}(z)=-\frac{a_{k+1}}{2 i \sin z}\left\{\Delta e_{k+1}(-z)[\right. & \left.\alpha P_{k-1}(z)+\beta \nabla P_{k-1}(z)\right] \\
- & \left.e_{k+1}(-z)\left[\gamma P_{k-1}(z)+\delta \nabla P_{k-1}(z)\right]\right\} .
\end{aligned}
$$

Hence, it is obvious to calculate

$$
\begin{array}{lll}
\text { (i) } W\left[E_{n}(z), F_{n}(z)\right]=\frac{M_{22}}{\operatorname{det} M}, & n=0,1,2, \ldots, k-1, \\
\text { (ii) } W\left[E_{n}(z), F_{n}(z)\right]=-2 i M_{22} \sin z, & & n=k+1, k+2, \ldots
\end{array}
$$

for all $z \in\left[-\frac{\pi}{2}, \frac{3 \pi}{2}\right] \backslash\{0, \pi\}$. 


\section{Resolvent operator And COntinuous spectrum}

Let us introduce the impulsive difference operator $L$ generated by the impulsive difference boundary value problem $(3)-(5)$ in the Hilbert space $\ell^{2}(\mathbb{N})$ such that

$$
\ell^{2}(\mathbb{N}):=\left\{y=\left\{y_{n}\right\}_{n \in \mathbb{N}}, y_{n} \in \mathbb{C},|y|^{2}=\sum_{n \in \mathbb{N}}\left|y_{n}\right|^{2}<\infty\right\} .
$$

We shall define two semi strips

$$
S_{0}:=\left\{z: z=\eta+i \xi,-\frac{\pi}{2} \leq \eta \leq \frac{3 \pi}{2}, \xi>0\right\}
$$

and

$$
S:=S_{0} \cup\left[-\frac{\pi}{2}, \frac{3 \pi}{2}\right]
$$

In view of $(9)$, it is obvious that $E_{n}(z)$ has an analytic continuation from $\left[-\frac{\pi}{2}, \frac{3 \pi}{2}\right]$ to $S_{0}$ and continuous up to $\left[-\frac{\pi}{2}, \frac{3 \pi}{2}\right]$ because of analytic properties of $Q_{n}(z), P_{n}(z)$ and $e_{n}(z)$. Thus, $F_{n}(z)$ turns into

$$
\widehat{F}_{n}(z)=\left\{\begin{array}{c}
P_{n}(z), \quad n=0,1,2, \ldots, k-1 \\
\widehat{M}_{12}(z) e_{n}(z)+M_{22}(z) \widehat{e}_{n}(z), \quad n=k+1, k+2, \ldots
\end{array},\right.
$$

where $\widehat{e}_{n}(z)$ is the unbounded solution of $(3)$, satisfying the condition

$$
\lim _{n \rightarrow \infty} e^{i n z} \widehat{e}_{n}(z)=1, \quad z \in \overline{\mathbb{C}}_{+}
$$

for $n=k+1, k+2, \ldots$.

We remark here that under the analytic continuation, function $M_{22}$ remains unchanged, whereas $M_{12}$ turns into

$$
\begin{aligned}
\widehat{M}_{12}(z)=-\frac{a_{k+1}}{2 i \sin z}\left\{\Delta \widehat{e}_{k+1}(z)\right. & {\left[\alpha P_{k-1}(z)+\beta \nabla P_{k-1}(z)\right] } \\
& \left.-\widehat{e}_{k+1}(z)\left[\gamma P_{k-1}(z)+\delta \nabla P_{k-1}(z)\right]\right\} .
\end{aligned}
$$

Lemma 1. The following equations hold for all $z \in S \backslash\{0, \pi\}$.

$$
\begin{gathered}
\text { (i) } W\left[\widehat{F}_{n}(z), E_{n}(z)\right]=-\frac{M_{22}(z)}{\operatorname{det} M}, \quad n \rightarrow 0, \\
\text { (ii) } W\left[\widehat{F}_{n}(z), E_{n}(z)\right]=2 i M_{22}(z) \sin z, \quad n \rightarrow \infty .
\end{gathered}
$$

Proof. Recall that Wronskian of any two solutions $y=\left\{y_{n}\right\}_{n \in \mathbb{N}}$ and $u=\left\{u_{n}\right\}_{n \in \mathbb{N}}$ of $(3)$ is defined as

$$
W[y, u]:=a_{n}\left[y_{n} u_{n+1}-y_{n+1} u_{n}\right] .
$$


Then, due to the fact that

$$
W\left[e_{n}(z), \widehat{e}_{n}(z)\right]=-2 i \sin z, \quad z \in \overline{\mathbb{C}}_{+},
$$

it is obvious to calculate Wronskian of the solutions $\widehat{F}_{n}$ and $E_{n}$.

In view of these solutions, we can compute the resolvent operator of $L$.

\section{Theorem 2.}

$$
\left(\mathcal{R}_{\lambda}(L) \psi\right)_{n}:=\sum_{m \in \mathbb{N}} \mathcal{G}_{n, m}(z) \psi_{m}, \quad \psi:=\left\{\psi_{m}\right\}_{m \in \mathbb{N}} \in \ell^{2}(\mathbb{N}), n \in \mathbb{N},
$$

is the resolvent operator of $L$, where

$$
\mathcal{G}_{n, m}(z)= \begin{cases}\frac{E_{n}(z) \widehat{F}_{m}(z)}{W\left[\widehat{F}_{n}(z), E_{n}(z)\right]}, & m=0,1,2, \ldots, n-1 \\ \frac{E_{m}(z) \widehat{F}_{n}(z)}{W\left[\widehat{F}_{n}(z), E_{n}(z)\right]}, & m=n, n+1, \ldots\end{cases}
$$

is defined as Green function for $z \in S$ and $m, n \neq k$.

Proof. In order to get the resolvent operator, we need to find the general solution of the equation

$$
a_{n-1} y_{n-1}+b_{n} y_{n}+a_{n} y_{n+1}-\lambda y_{n}=\psi_{n}, \quad n \in \mathbb{N} \backslash\{k-1, k, k+1\},
$$

where $\psi_{n} \in \ell^{2}(\mathbb{N})$. For this reason, we use the solutions $\widehat{F}_{n}$ and $E_{n}$ to write the general solution of the homogenous part of (15). So, by the help of variation of parameters method and some iterations, we get Green function and resolvent operator of L.

Theorem 3. Assuming that the condition (6) satisfies, then $\sigma_{c}(L)=[-2,2]$, where $\sigma_{c}(L)$ denotes the continuous spectrum of $L$.

Proof. In order to prove to this theorem, we first need to introduce the difference operators $L_{0}$ and $L_{1}$ generated by the following difference expressions in $\ell^{2}(\mathbb{N})$ together with (5)

$$
\begin{aligned}
& \left(\ell_{0} y\right)_{n}=y_{n-1}+y_{n+1}, \quad n \in \mathbb{N} \backslash\{k-1, k+1\} \\
& \left(\ell_{1} y\right)_{n}=\left(a_{n-1}-1\right) y_{n-1}+b_{n} y_{n}+\left(a_{n}-1\right) y_{n+1}, \quad n \in \mathbb{N} \backslash\{k-1, k, k+1\},
\end{aligned}
$$

respectively. It is evident that $L_{0}$ is not selfadjoint but it can be written as the sum of a selfadjoint and a finite dimensional operator in $\ell^{2}(\mathbb{N})$. On the other hand, $L_{1}$ is a compact operator [21]. Since all finite dimensional operators are compact, the impulsive operator $L$ can be represented as the sum of a selfadjoint and two compact operators. By Weyl theorem of a compact perturbation [22], the continuous spectrum of $L$ coincides with the continuous spectrum of the selfadjoint operator which is $[-2,2]$. So, the proof is completed. 


\section{MAIN RESUlTS}

In this section, we determine the sets of eigenvalues and spectral singularities and discuss their numerical properties.

From the definition of eigenvalues and (14), we introduce the set of eigenvalues of impulsive operator $L$ as

$$
\sigma_{d}(L)=\left\{\lambda \in \mathbb{C}: \lambda=2 \cos z, z \in S_{0}, M_{22}(z)=0\right\} .
$$

Spectral singularities are defined as the poles of the kernel of resolvent operator and are also embedded in the continuous spectrum. Hence, we have

$$
\sigma_{s s}(L)=\left\{\lambda \in \mathbb{C}: \lambda=2 \cos z, z \in\left[-\frac{\pi}{2}, \frac{3 \pi}{2}\right] \backslash\{0, \pi\}, M_{22}(z)=0\right\},
$$

where $\sigma_{s s}(L)$ denotes the set of spectral singularities of $L$.

To study numerical properties of the sets $\sigma_{d}(L)$ and $\sigma_{s s}(L)$, we need to examine the numerical properties of the zeros of $M_{22}$ in $S$. For this reason, we define the sets

$$
\begin{gathered}
S_{1}:=\left\{z: z \in S_{0}, M_{22}(z)=0\right\}, \\
S_{2}:=\left\{z: z \in\left[-\frac{\pi}{2}, \frac{3 \pi}{2}\right], M_{22}(z)=0\right\} .
\end{gathered}
$$

To prove the next lemma and theorem, we need the following theorem given in 20]:

Theorem 4. Under the condition (6), the function $M_{22}$ satisfies the following asymptotics for $\xi \rightarrow \infty$, where $z=\eta+i \xi$,

(i) If $\alpha+\beta+\gamma+\delta \neq 0$,

$$
M_{22}=e^{4 i z}\left(\prod_{n=1}^{k-2} a_{n}\right)^{-1}\left[(\alpha+\beta+\gamma+\delta) \mu_{k+1}+o(1)\right] .
$$

(ii) If $\alpha+\beta+\gamma+\delta=0$,

$$
M_{22}=e^{5 i z}\left(\prod_{n=1}^{k-3} a_{n}\right)^{-1}\left[-a_{k-2}^{-1}(\alpha+\beta) \mu_{k+2}-(\beta+\delta) \mu_{k+1}+o(1)\right] .
$$

Lemma 5. Assume (6).

(i) The set $S_{1}$ is bounded, is no more than countable number of elements and its limit points can lie only in $\left[-\frac{\pi}{2}, \frac{3 \pi}{2}\right]$.

(ii) The set $S_{2}$ is compact and its linear Lebesgue measure is zero.

Proof. (i) Theorem 4 proves that $M_{22}$ cannot equal to zero for sufficiently large $\lambda \in \bar{C}_{+}$. Thus, the boundedness of the sets $S_{1}$ and $S_{2}$ is clear from Theorem 4 . Moreover, since $M_{22}$ is analytic in $\mathbb{C}_{+}$, the set $S_{1}$ has at most countable number of elements, and its limit points can only lie in $\left[-\frac{\pi}{2}, \frac{3 \pi}{2}\right]$. 
(ii) Using uniqueness theorem of analytic functions [23], we obtain that $S_{2}$ is a closed set and its linear Lebesgue measure is zero.

We can give following theorem as a direct consequence of $(16),(17)$ and Lemma 5.

Theorem 6. Under the condition (6),

(i) the set of eigenvalues of $L$ is bounded and countable, its limit points can lie only in $[-2,2]$,

(ii) the set of spectral singularities of $L$ is compact and its linear Lebesgue measure is zero.

Definition 7. The multiplicity of a zero of $M_{22}$ in $S$ is called the multiplicity of corresponding eigenvalue or spectral singularity of impulsive operator $L$.

Theorem 8. If

$$
\sup _{n \in \mathbb{N}}\left\{e^{\epsilon n}\left(\left|1-a_{n}\right|+\left|b_{n}\right|\right)\right\}<\infty
$$

for some $\epsilon>0$, then there are finitely number of eigenvalues and spectral singularities of the operator $L$, and each of them has finite multiplicity.

Proof. Under the condition (20), it follows from (8) that

$$
\left|A_{j, m}\right| \leq c e^{-\frac{\epsilon}{4} m}, \quad j=k+1, k+2 ; \quad m=1,2, \ldots,
$$

where $c$ is an arbitrary constant. From (11), $M_{22}(z)$ has following representation

$$
\begin{aligned}
M_{22}(z)= & -\frac{a_{k+1}}{2 i \sin z}\left\{-(\alpha+\beta) P_{k-1}(z) \mu_{k+2} e^{i(k+2) z}\left(1+\sum_{m=1}^{\infty} A_{k+2, m} e^{i m z}\right)\right. \\
& +\beta P_{k-2}(z) \mu_{k+2} e^{i(k+2) z}\left(1+\sum_{m=1}^{\infty} A_{k+2, m} e^{i m z}\right) \\
+ & (\alpha+\beta+\gamma+\delta) P_{k-1}(z) \mu_{k+1} e^{i(k+1) z}\left(1+\sum_{m=1}^{\infty} A_{k+1, m} e^{i m z}\right) \\
& \left.-(\beta+\delta) P_{k-2}(z) \mu_{k+1} e^{i(k+1) z}\left(1+\sum_{m=1}^{\infty} A_{k+1, m} e^{i m z}\right)\right\} .
\end{aligned}
$$

By the help of (21) and (22), we conclude that $M_{22}$ has an analytic continuation to the half plane $\operatorname{Im} z>-\frac{\epsilon}{4}$. Hence, the sets $\sigma_{d}(L)$ and $\sigma_{s s}(L)$ have no limit points in $\left[-\frac{\pi}{2}, \frac{3 \pi}{2}\right]$. By Theorem 6 , we find that these sets are bounded and have a finite number of elements. Finally, using uniqueness theorem of analytic functions, we see that all zeros of $M_{22}$ in $S$ have finite multiplicities. 
Let us denote the sets of all limit points of $S_{1}$ and $S_{2}$ by $S_{3}$ and $S_{4}$, respectively and the set of all zeros of $M_{22}$ with infinite multiplicity in $S$ by $S_{5}$. From the uniqueness theorem of analytic functions, we find that

$$
S_{1} \cap S_{5}=\emptyset, \quad S_{3} \subset S_{2}, \quad S_{4} \subset S_{2}, \quad S_{5} \subset S_{2} \quad S_{3} \subset S_{5}, \quad S_{4} \subset S_{5}
$$

and

$$
\mu\left(S_{3}\right)=\mu\left(S_{4}\right)=\mu\left(S_{5}\right)=0 .
$$

Now, for some $\epsilon>0$ and $\frac{1}{2} \leq \rho<1$, let us consider the condition

$$
\sup _{n \in \mathbb{N}}\left\{e^{\epsilon n^{\rho}}\left(\left|1-a_{n}\right|+\left|b_{n}\right|\right)\right\}<\infty,
$$

which is weaker than $(20)$. Under the condition 23 , the function $M_{22}$ cannot be continued analytically from $\left[-\frac{\pi}{2}, \frac{3 \pi}{2}\right]$ to the lower half plane. So, we need some preliminaries before giving the main result.

For the sake of simplicity, let us define

$$
H(z):=M_{22}(z) 2 i \sin z,
$$

which is also analytic in $\mathbb{C}_{+}$and infinitely differentiable on real axis.

Lemma 9. Under the condition (23), following inequality holds:

$$
\left|H^{(n)}(z)\right| \leq A_{n}
$$

for $z \in S$ and $n=0,1, \ldots$, where

$$
A_{n} \leq \widetilde{C} 2^{n} K b^{k} k ! k^{k \frac{1-\rho}{\rho}},
$$

$\widetilde{C}, K, b$ are positive constants depending on $\epsilon$ and $\rho$.

Proof. From 22, we can write

$$
\begin{aligned}
& H(z)=-a_{k+1}\left\{-(\alpha+\beta) \mu_{k+2} e^{i(k+2) z}\left(1+\sum_{m=1}^{\infty} A_{k+2, m} e^{i m z}\right)\right. \\
& \times\left(\frac{\left(e^{i z}+e^{-i z}\right)^{k-2}}{\prod_{i=1}^{k-2} a_{i}}+p^{k-3}(z)\right) \\
& +\beta \mu_{k+2} e^{i(k+2) z}\left(1+\sum_{m=1}^{\infty} A_{k+2, m} e^{i m z}\right)\left(\frac{\left(e^{i z}+e^{-i z}\right)^{k-3}}{\prod_{i=1}^{k-3} a_{i}}+p^{k-4}(z)\right)
\end{aligned}
$$




$$
\begin{aligned}
& +(\alpha+\beta+\gamma+\delta) \mu_{k+1} e^{i(k+1) z}\left(1+\sum_{m=1}^{\infty} A_{k+1, m} e^{i m z}\right) \\
& \times\left(\frac{\left(e^{i z}+e^{-i z}\right)^{k-2}}{\prod_{i=1}^{k-2} a_{i}}+p^{k-3}(z)\right) \\
& -(\beta+\delta) \mu_{k+1} e^{i(k+1) z}\left(1+\sum_{m=1}^{\infty} A_{k+1, m} e^{i m z}\right) \\
& \left.\times\left\{\frac{\left(e^{i z}+e^{-i z}\right)^{k-3}}{\prod_{i=1}^{k-3} a_{i}}+p^{k-4}(z)\right)\right\},
\end{aligned}
$$

where the polynomial function $p^{k}(z)$ is of $k$-th degree. Moreover, by direct computation, we find

$$
\begin{aligned}
\left|H^{(n)}(z)\right| \leq & \left|a_{k+1}\right|\left\{\left|(\alpha+\beta) \mu_{k+2}\right| \frac{C}{\left|\prod_{i=1}^{k-2} a_{i}\right|} \sum_{s=0}^{n}\left(\begin{array}{c}
n \\
s
\end{array}\right)\left(\sum_{m=1}^{\infty}\left|A_{k+2, m}\right| m^{k-s}\right)(2 k)^{s}\right. \\
& +\left|\beta \mu_{k+2}\right| \frac{C}{\left|\prod_{i=1}^{k-3} a_{i}\right|} \sum_{s=0}^{n}\left(\begin{array}{c}
n \\
s
\end{array}\right)\left(\sum_{m=1}^{\infty}\left|A_{k+2, m}\right| m^{k-s}\right)(2 k-1)^{s} \\
& +\left|(\alpha+\beta+\gamma+\delta) \mu_{k+1}\right| \frac{C}{\left|\prod_{i=1}^{k-2} a_{i}\right|} \sum_{s=0}^{n}\left(\begin{array}{c}
n \\
s
\end{array}\right)\left(\sum_{m=1}^{\infty}\left|A_{k+1, m}\right| m^{k-s}\right)(2 k-1)^{s} \\
& \left.+\left|(\beta+\delta) \mu_{k+1}\right| \frac{C}{\left|\prod_{i=1}^{k-3} a_{i}\right|} \sum_{s=0}^{n}\left(\begin{array}{c}
n \\
s
\end{array}\right)\left(\sum_{m=1}^{\infty}\left|A_{k+1, m}\right| m^{k-s}\right)[2(k-1)]^{s}\right\}
\end{aligned}
$$


In accordance with 8 and 23, , we get

$$
\left|A_{j, m}\right| \leq c_{1} e^{-\frac{\epsilon}{2}\left(\frac{m}{2}\right)^{\rho}}, \quad j=k+1, k+2 ; \quad m=1,2, \ldots,
$$

where $c_{1}$ is an arbitrary positive constant. Therefore, by (26), we arrive at

$$
\left|H^{(n)}(z)\right| \leq K\left|a_{k+1}\right| 2^{n} \widetilde{C} \sum_{m=1}^{\infty} e^{-\frac{\epsilon}{2}\left(\frac{m}{2}\right)^{\rho}} m^{k}
$$

where

$$
\begin{aligned}
K: & =\left\{\left|(\alpha+\beta) \mu_{k+2}\right|\left(\prod_{i=1}^{k-2} a_{i}\right)^{-1}+\left|\beta \mu_{k+2}\right|\left(\prod_{i=1}^{k-3} a_{i}\right)^{-1}\right. \\
& \left.+\left|(\alpha+\beta+\gamma+\delta) \mu_{k+1}\right|\left(\prod_{i=1}^{k-2} a_{i}\right)^{-1}+\left|(\beta+\delta) \mu_{k+1}\right|\left(\prod_{i=1}^{k-3} a_{i}\right)^{-1}\right\} .
\end{aligned}
$$

On the other hand, if we define

$$
D_{k}:=\sum_{m=1}^{\infty} e^{-\frac{\epsilon}{2}\left(\frac{m}{2}\right)^{\rho}} m^{k}
$$

by the help of Gamma function we estimate

$$
D_{k}=\int_{0}^{\infty} t^{k} e^{-\frac{\epsilon}{2}\left(\frac{t}{2}\right)^{\rho}} d t=\frac{2^{k+1+\frac{k+1}{\rho}}}{\rho \epsilon^{\frac{k+1}{\rho}}}\left(\frac{k+1}{\rho}-1\right) \Gamma\left(\frac{k+1}{\rho}-1\right) .
$$

After that, using the inequalities $1+\frac{1}{k} \leq e$ and $k^{k} \leq e^{k} k$ ! for $k \in \mathbb{N}$, we get

$$
D_{k} \leq b^{k} K k ! k^{k \frac{1-\rho}{\rho}}
$$

which gives the proof of the lemma.

Theorem 10. Under the condition (23), $S_{5}=\emptyset$.

Proof. Since the function $M_{22}$ is not equal to zero identically, according to [15], we obtain

$$
\int_{0}^{w} \ln T(s) d \mu\left(S_{5}, s\right)>-\infty
$$

where

$$
T(s)=\inf _{k} \frac{H_{k} s^{k}}{k !}, \quad k \in \mathbb{N} \cup\{0\},
$$

$\mu\left(S_{5}, s\right)$ denotes the Lebesque measure of $s$-neighbourhood of $S_{5}$ and $H_{k}$ is defined by Lemma 9 . Using Lemma 9, we calculate 


$$
T(s) \leq K \exp \left\{-\frac{1-\rho}{\rho} e^{-1} b^{-\frac{\rho}{1-\rho}} s^{-\frac{\rho}{1-\rho}}\right\} .
$$

Hence, we see from (27) that

$$
\int_{0}^{w} s^{-\frac{\delta}{1-\delta}} d \mu\left(S_{5}, s\right) \leq-\int_{0}^{w} \ln T(s) d \mu\left(S_{5}, s\right)<\infty .
$$

Since $\frac{\rho}{1-\rho} \geq 1$, the integral on the left handside is convergent for arbitrary $s$ if and only if $\mu\left(S_{5}, s\right)=0$, i.e., $S_{5}=\emptyset$.

\section{PRincipal FunCtions}

In this section, we determine the principal functions of impulsive operator $L$. Since, $\lambda=2 \cos z$ transforms the semi strip $S_{0}$ to the set $\Omega:=\mathbb{C} \backslash[-2,2]$. We shall define the functions

$$
\begin{aligned}
\widetilde{F}_{n}(\lambda): & =\widehat{F}_{n}\left(\arccos \frac{\lambda}{2}\right), \quad n \in \mathbb{N} \backslash\{k\}, \\
\widetilde{E}_{n}(\lambda): & =E_{n}\left(\arccos \frac{\lambda}{2}\right), \quad n \in \mathbb{N} \backslash\{k\}, \\
\widetilde{M}_{22}(\lambda): & =M_{22}\left(\arccos \frac{\lambda}{2}\right) .
\end{aligned}
$$

Obviously, $\widetilde{F}_{n}(\lambda)$ and $\widetilde{E}_{n}(\lambda)$ are solutions of (3)-(5). By (16) and (17), we obtain that

$$
\begin{aligned}
\sigma_{d}(L) & =\left\{\lambda: \lambda \in \Omega, \widetilde{M}_{22}(\lambda)=0\right\} \\
\sigma_{s s}(L) & =\left\{\lambda: \lambda \in[-2,2], \widetilde{M}_{22}(\lambda)=0\right\} .
\end{aligned}
$$

Moreover, condition (23) guarentees finiteness of zeros $\widetilde{M}_{22}$ in $\Omega$ and in [-2,2]. Let $\lambda_{1}, \lambda_{2}, \ldots, \lambda_{p}$ denote the zeros of the function $\widetilde{M}_{22}$ in $\Omega$ with multiplicities $m_{1}, m_{2}, \ldots, m_{p}$, respectively. Similarly, let $\lambda_{p+1}, \lambda_{p+2}, \ldots \lambda_{t}$ be zeros of $\widetilde{M}_{22}$ in $[-2,2]$ with multiplicities $m_{p+1}, m_{p+2}, \ldots, m_{t}$, respectively. Thus,

$$
\left\{\frac{d^{s}}{d \lambda^{s}} W\left[\widetilde{F}_{n}(\lambda), \widetilde{E}_{n}(\lambda)\right]\right\}_{\lambda=\lambda_{j}}=\left\{\frac{d^{s}}{d \lambda^{s}} \widetilde{M}_{22}(\lambda)\right\}_{\lambda=\lambda_{j}}=0
$$

holds for $s=0,1, \ldots, m_{j}-1, j=1,2, \ldots, p, p+1, \ldots, t$.

Theorem 11.

$$
\left\{\frac{d^{s}}{d \lambda^{s}} \widetilde{F}_{n}(\lambda)\right\}_{\lambda=\lambda_{j}}=\sum_{r=0}^{s}\left(\begin{array}{c}
s \\
r
\end{array}\right) A_{r}\left(\lambda_{j}\right)\left\{\frac{d^{s}}{d \lambda^{s}} \widetilde{E}_{n}(\lambda)\right\}_{\lambda=\lambda_{j}}
$$

holds for $s=0,1, \ldots, m_{j}-1, j=1,2, \ldots, p, p+1, \ldots, t$. 
Proof. We will continue by mathematical induction. Let $s=0$. From 28, we find

$$
\widetilde{F}_{n}\left(\lambda_{j}\right)=A_{o}\left(\lambda_{j}\right) \widetilde{E}_{n}\left(\lambda_{j}\right), \quad n \in \mathbb{N},
$$

where $A_{o}\left(\lambda_{j}\right) \neq 0$. Let us assume that

$$
\left\{\frac{d^{s_{0}}}{d \lambda^{s_{0}}} \widetilde{F}_{n}(\lambda)\right\}_{\lambda=\lambda_{j}}=\sum_{r=0}^{s_{0}}\left(\begin{array}{c}
s_{0} \\
r
\end{array}\right) A_{r}\left(\lambda_{j}\right)\left\{\frac{d^{s_{0}}}{d \lambda^{s_{0}}} \widetilde{E}_{n}(\lambda)\right\}_{\lambda=\lambda_{j}}
$$

holds for $1 \leq s_{0} \leq m_{j}-2$. If $\left\{y_{n}(\lambda)\right\}_{n \in \mathbb{N}}$ is a solution of (3), then we obtain

$$
a_{n-1} \frac{d^{s}}{d \lambda^{s}} y_{n-1}(\lambda)+b_{n} \frac{d^{s}}{d \lambda^{s}} y_{n}(\lambda)+a_{n} \frac{d^{s}}{d \lambda^{s}} y_{n+1}(\lambda)-\lambda \frac{d^{s}}{d \lambda^{s}} y_{n}(\lambda)=s \frac{d^{s-1}}{d \lambda^{s-1}} y_{n}(\lambda) .
$$

Writing last equality for solutions $\widetilde{F}_{n}\left(\lambda_{j}\right)$ and $\widetilde{E}_{n}\left(\lambda_{j}\right)$ then, using (28) and (30), we find that 29p holds for $s=0,1, \ldots, m_{j}-1, j=1,2, \ldots, p, p+1, \ldots, t$.

Using the notation

$$
\mathcal{A}_{r}\left(\lambda_{j}\right):=\frac{A_{r}\left(\lambda_{j}\right)}{(s-r) !}
$$

we can write 29$)$ as

$$
\frac{1}{s !}\left\{\frac{d^{s}}{d \lambda^{s}} \widetilde{F}_{n}(\lambda)\right\}_{\lambda=\lambda_{j}}=\sum_{r=0}^{s}\left(\begin{array}{c}
s \\
r
\end{array}\right) \mathcal{A}_{r}\left(\lambda_{j}\right) \frac{1}{r !}\left\{\frac{d^{s}}{d \lambda^{s}} \widetilde{E}_{n}(\lambda)\right\}_{\lambda=\lambda_{j}}
$$

for $s=0,1, \ldots, m_{j}-1, j=1,2, \ldots, p, p+1, \ldots, t$.

Now, let us introduce the functions

$$
U^{(s)}\left(\lambda_{j}\right)=\left\{U_{n}^{(s)}\left(\lambda_{j}\right)\right\}_{n \in \mathbb{N} \backslash\{k\}}, \quad s=0,1, \ldots, m_{j}-1, j=1,2, \ldots, t,
$$

where

$$
\begin{aligned}
U_{n}^{(s)}\left(\lambda_{j}\right) & =\frac{1}{s !}\left\{\frac{d^{s}}{d \lambda^{s}} \widetilde{F}_{n}(\lambda)\right\}_{\lambda=\lambda_{j}} \\
& =\sum_{r=0}^{s}\left(\begin{array}{c}
s \\
r
\end{array}\right) \mathcal{A}_{r}\left(\lambda_{j}\right) \frac{1}{r !}\left\{\frac{d^{s}}{d \lambda^{s}} \widetilde{E}_{n}(\lambda)\right\}_{\lambda=\lambda_{j}} .
\end{aligned}
$$

The functions $U^{(s)}\left(\lambda_{j}\right), s=0,1, \ldots, m_{j}-1, j=1,2, \ldots, p$ and $U^{(s)}\left(\lambda_{j}\right)$, $s=0,1, \ldots, m_{j}-1, j=p+1, p+2, \ldots, t$ are called the principal functions of eigenvalues and spectral singularities of impulsive operator $L$, respectively.

In view of the properties of principal functions of corresponding operator, we easily get the following theorem.

\section{Theorem 12.}

$$
\begin{aligned}
& U^{(s)}\left(\lambda_{j}\right) \in \ell^{2}(\mathbb{N}), \quad s=0,1, \ldots, m_{j}-1, j=1,2, \ldots, p . \\
& U^{(s)}\left(\lambda_{j}\right) \notin \ell^{2}(\mathbb{N}), \quad s=0,1, \ldots, m_{j}-1, j=p+1, p+2, \ldots, t .
\end{aligned}
$$




\section{REFERENCES}

[1] Samoilenko, A. M. and Perestyuk, N. A., Impulsive differential equations, World Scientific, Singapore, 1995.

[2] Lakshmikantham, V., Bainov, D. D. and Simeonov, P. S., Theory of impulsive differential equations, World Scientific, Singapore, 1998.

[3] Bainov, D. D. and Simeonov, P. S., Oscillation theory of impulsive differential equations, Int. Publ., Orlando, 1998.

[4] Uğurlu, E. and Bairamov, E., Spectral analysis of eigenparameter dependent boundary value transmission problems, J. Math. Anal. Appl., 413, 1, (2014), 482-494.

[5] Mostafazadeh, A., Spectral singularities of a general point interaction, J. Phys. A. Math. Theory, 44, 375302, (2011), (9pp).

[6] He, Z. M. and Zhang, X. M., Monoton iterative technique for first order impulsive difference equations with periodic boundary conditions, Appl. Math. Comput., 156, 3, (2004), 605-620.

[7] Wang, P. and Wang, W., Boundary value problems for first order impulsive difference equations, Int. Journal of Difference Equations, 1, (2006), 249-259.

[8] Zhang, Q., On a linear delay difference equations with impulses., Annals of Differential Equations, 18, 2, (2002), 197-204.

[9] Naimark, M. A., Investigation of the spectrum and the expansion in eigenfunctions of a nonselfadjoint operators of second order on a semi-axis, AMS Transl. (2), 16, (1960), 103-193.

[10] Marchenko, V. A., Sturm-Liouville operators and applications, Birkhauser Verlag, Basel, 1986.

[11] Levitan B. M. and Sargsjan I. S., Sturm-Liouville and Dirac operators, Kluwer Academic Publishers Group, Dordrecht, 1991.

[12] Agarwal, R. P., Difference equations and inequalities, in: Theory, Methods and Applications, Marcel Dekkar Inc., New York, Basel, 2000.

[13] Kelley, W. G and Peterson, A. C., Difference equations: an introduction with applications, Harcourt Academic Press, 2001.

[14] Krall, A.M., Bairamov, E. and Cakar, O., Spectral analysis of a non-selfadjoint discrete Schrödinger operators with spectral singularities, Math. Nachr., 231, (2001), 89-104.

[15] Bairamov, E., Cakar, O. and Krall, A.M., Non-Selfadjoint Difference Operators and Jacobi Matrices with Spectral Singularities, Math. Nachr., 229, (2001), 5-14.

[16] Adıvar, M. and Bairamov, E., Spectral Properties of Non-Selfadjoint Difference Operators, J. Math. Anal. and Appl., 261, (2001), 461-478.

[17] Adivar, M. and Bairamov, E., Difference Equations of Second Order with Spectral Singularities, J. Math. Anal. Appl., 277, (2003), 714-721.

[18] Guseinov, G. Sh., The inverse problem of scattering theory for a second order difference equation, Sov. Math., Dokl., 230, (1976), 1045-1048.

[19] Olgun, M., Köprübaşı, T. and Aygar, Y., Principal functions of non-selfadjoint difference operator with spectral parameter in boundary conditions, Abstr. Appl. Anal., Art. ID 608329, 10, (2011).

[20] Erdal, I., Yardımcı, S., Eigenvalues and Scattering Properties of Difference Operators with Impulsive Condition, Commun. Fac. Sci. Univ. Ank. Ser. A1 Math. Stat., 68, 1, (2019), 663-671.

[21] Lusternik, L.A, Sobolev V. I., Elements of Functional Analysis, Halsted Press, New York, 1974.

[22] Glazman I. M., Direct Methods of Qualitative Spectral Anaysis of Singular Differential Operators, Jerusalem, 1965.

[23] Dolzhenko E. P., Boundary value uniqueness theorems for analytic functions, Math. Notes, $26,(1979), 437-442$. 
Current address: Şeyhmus Yardımcı: Ankara University, Faculty of Science, Department of Mathematics, 06100, Ankara, Turkey.

E-mail address: smyardimci@ankara.edu.tr

ORCID Address: http://orcid.org/0000-0002-1062-9000

Current address: Ibrahim Erdal: Ankara University, Faculty of Science, Department of Mathematics, 06100, Ankara, Turkey.

E-mail address: ierdal@ankara.edu.tr

ORCID Address: http://orcid.org/0000-0002-4445-2389 